\title{
A network topology approach to diagnose the shift of covariance structure
}

\author{
Nur Syahidah Yusoff ${ }^{1,}{ }^{*}$, Noryanti Mohamad ${ }^{1}$, Chuan Zun Liang ${ }^{1}$, Shamshuritawati Sharif ${ }^{2}$, \\ and Tan Lit $\mathrm{Ken}^{3}$ \\ ${ }^{1}$ Faculty of Industrial Sciences \& Technology, Universiti Malaysia Pahang, 26300, Gambang,Pahang, \\ Malaysia \\ ${ }^{2}$ School of Quantitative Sciences, UUM-College Arts and Science, Universiti Utara Malaysia, Sintok, \\ Kedah, Malaysia \\ ${ }^{3}$ Department of Mechanical Precision Engineering, Malaysia-Japan International Institute of \\ Technology (MJIIT), Universiti Teknologi Malaysia Kuala Lumpur, Jalan Sultan Yahya Petra, 54100, \\ Kuala Lumpur, Malaysia
}

\begin{abstract}
Understanding the shift of covariance matrices in any process is not an easy task. From the literatures, the most popular and widely used test for covariance shift is Jennrich's test and Box's M test. It is important to note that Box and also Jennrich have constructed their own test by involving sample covariance matrix determinant or, equivalently, generalized variance $(\mathrm{GV})$ as multivariate variability measure. However, $\mathrm{GV}$ has serious limitations as a multivariate variability measure. Those limitations of GV motivate us to use a proposed test based on an alternative measure of multivariate variability called vector variance (VV). However, if after hypothesis testing the hypothesis of stable process covariance is rejected, then the next problem is to find the cause of that situation. In this paper, network topology approach will be used to understand the shift. A case study will be discussed and presented to illustrate the advantage of this approach.
\end{abstract}

\section{Introduction}

The shift of the covariance structure can be determined by testing the stability of covariance structure. The importance of covariance structure stability has been shown in many areas. For example, in financial market [1-3], real estate industry [4], service industry [5, 6], manufacturing industry $[7,8]$, software industry $[9,10]$, health care industry $[11]$ and even supply chain management [12].

To test the stability of covariance structure, we use a test proposed by Yusoff and Djauhari [13]. In this study, if the null hypothesis about the stability of covariance structure is rejected, we use the network analysis approach to find out the root causes of that rejection. For that purpose, covariance matrix will be considered as representing a network. To filter the information in that network, we use minimum spanning tree (MST) issued

\footnotetext{
*Corresponding author: wnsyahidah@ump.edu.my
} 
from Kruskal's algorithm. Then, we construct the network topology of all variables. Its interpretation will be delivered by using centrality measures such as degree centrality and betweenness centrality.

The rest of the paper is organised as follows. In the Section 2, we present the related methodology, followed by results and discussion of corresponding example in Section 3. At the end, this paper is closed with a conclusion in the last section.

\section{Methodology}

In this section we focus on the shift of the covariance structure, i.e. testing the equality of two covariance matrices. However, to understand the shift of covariance is not an easy task. The complexity of multivariate data analysis, and the cross correlation among currencies make it difficult for the analysis. This motivate us to introduce a covariance network topology as a further analysis if there exists a shift in covariance structure, otherwise the analysis is not required.

\subsection{Case study: Currency exchange rate}

In this study, as an illustrative example, covariance matrices of foreign exchange rate time series are analyzed for 78 world currencies (European Euro Analysis), retrieved from Pacific Exchange Rate Service website. Those data were retrieved from January 2000 until January 2010 and they were analyzed quarterly. Hence, there are 40 corresponding covariance matrices in this study, i.e., S1, S2, .., S40.

\subsection{Yusoff \& Djauhari's test}

Proposed statistical test by Yusoff and Djauhari [13] used VV as the multivariate variability measure as an alternative measure to substitute GV. The limitations of GV as the multivariate variability measure can be seen in [13] and [14]. VV is a sum squared of the elements of sample covariance matrix, i.e., $\operatorname{Tr}\left(S^{2}\right)$. By using Yusoff \& Djauhari's test (1) the hypotheses involved are $H_{0}: \Sigma_{i}=\Sigma_{0}$ versus $H_{1}: \Sigma_{i} \neq \Sigma_{0}$ with $i=1,2, \ldots, m$ and $\Sigma_{0}$; covariance matrix for the whole data. For $\alpha=0.05$, we obtain $Z_{1}, Z_{2}, \ldots, Z_{40}$ are as in table 1 with critical value, $z_{0.025}=1.96 . H_{0}$ is rejected if $|Z|>z_{0.025}$. Therefore, according to table 1, we conclude that $S_{1}, S_{2}, S_{3}, S_{4}, S_{10}, S_{11}, S_{12}, S_{15}, S_{16}, S_{18}, S_{19}, S_{20}, S_{21}, S_{22}, S_{24}, S_{26}, S_{27}$, $S_{30}, S_{33}, S_{34}, S_{35}, S_{36}, S_{37}$ and $S_{38}$ are not stable. In the next section, we will determine which variables are responsible for the instability of the covariance matrix. For that purpose, the average of the stable covariance matrices, $\bar{S}$ is obtained and it will be compared with the most unstable covariance matrix, $S_{35}$, i.e., the $Z$ statistic for $S_{35}$ is highest than the others.

$$
Z_{i}=\frac{\sqrt{n_{i}-1}\left(\operatorname{Tr}\left(S_{i}^{2}\right)-\operatorname{Tr}\left(\Sigma_{0}^{2}\right)\right)}{\sqrt{8 \operatorname{Tr}\left(\Sigma_{0}^{4}\right)}}
$$

distributed to $N(0,1)$ where

(i) $\operatorname{Tr}\left(\Sigma_{0}^{4}\right)=\sum_{i=1}^{p} \sum_{j=1}^{p}\left(\sigma_{i j}^{*}\right)^{2} ; \sigma_{i j}^{*}=$ elements $(i, j)$ of $\Sigma_{0}^{2}$ 
(ii) $\operatorname{Tr}\left(\Sigma_{0}^{2}\right)=\sum_{i=1}^{p} \sum_{j=1}^{p} \sigma_{i j}^{2} ; \sigma_{i j}=$ elements $(i, j)$ of $\Sigma_{0}$

(iii) $\operatorname{Tr}\left(S_{i}^{2}\right)=\sum_{i=1}^{p} \sum_{j=1}^{p} s_{i j}^{2} ; s_{i j}=$ elements $(i, j)$ of $S$

(iv) $n_{i}=$ sample size of covariance matrix.

\subsection{Network topology}

A network analysis will be used if unstable covariance matrix occurs, to find the most important variables which are responsible for that situation. For that purpose, first, we transform $S$ into a similarity matrices, $S^{*}$ by using one of the three methods for transforming $S$ into $S^{*}$ [15]. In this study, the first method of transforming $S$ into $S^{*}$ is used, i.e., $s_{i j}^{*}=\left|s_{i j}\right|$, see [16]. Then, we transform $S^{*}$ into dissimilarity matrices, $D$. Based on those dissimilarity matrices, by using Kruskal's algorithm provided in Matlab, we construct MST. From MST, we construct the network topology of all variables which will be visualized by using Pajek software. The interpretation of that network will be delivered by using centrality measures, i.e., degree centrality [17-18] and betweenness centrality [19-20].

Table 1. The value of $Z$ statistic.

\begin{tabular}{|c|c|c|c|c|c|c|c|}
\hline $\boldsymbol{m}$ & $\boldsymbol{Z}$ & $\mathbf{z}_{\mathbf{0 . 0 2 5}}$ & Result & $\boldsymbol{m}$ & $\mathbf{Z}$ & $\mathbf{z} 0.025$ & Result \\
\hline 1 & 13.7288 & 1.96 & reject $H_{0}$ & 21 & 2.6824 & 1.96 & reject $H_{0}$ \\
\hline 2 & 2.2158 & 1.96 & reject $H_{0}$ & 22 & 2.4410 & 1.96 & reject $H_{0}$ \\
\hline 3 & 2.3336 & 1.96 & reject $H_{0}$ & 23 & 1.8326 & 1.96 & do not reject $H_{0}$ \\
\hline 4 & 2.7082 & 1.96 & reject $H_{0}$ & 24 & 4.6534 & 1.96 & reject $H_{0}$ \\
\hline 5 & 1.9243 & 1.96 & do not reject $H_{0}$ & 25 & 0.3506 & 1.96 & do not reject $H_{0}$ \\
\hline 6 & 1.3824 & 1.96 & do not reject $H_{0}$ & 26 & 22.4887 & 1.96 & reject $H_{0}$ \\
\hline 7 & 1.1432 & 1.96 & do not reject $H_{0}$ & 27 & 4.2196 & 1.96 & reject $H_{0}$ \\
\hline 8 & 1.4454 & 1.96 & do not reject $H_{0}$ & 28 & 1.3561 & 1.96 & do not reject $H_{0}$ \\
\hline 9 & 1.4615 & 1.96 & do not reject $H_{0}$ & 29 & 1.4892 & 1.96 & do not reject $H_{0}$ \\
\hline 10 & 8.0879 & 1.96 & reject $H_{0}$ & 30 & 2.4128 & 1.96 & reject $H_{0}$ \\
\hline 11 & 2.5372 & 1.96 & reject $H_{0}$ & 31 & 1.8234 & 1.96 & do not reject $H_{0}$ \\
\hline 12 & 2.3465 & 1.96 & reject $H_{0}$ & 32 & 0.5625 & 1.96 & do not reject $H_{0}$ \\
\hline 13 & 1.3086 & 1.96 & do not reject $H_{0}$ & 33 & 45.9322 & 1.96 & reject $H_{0}$ \\
\hline 14 & 1.6130 & 1.96 & do not reject $H_{0}$ & 34 & 2.1058 & 1.96 & reject $H_{0}$ \\
\hline 15 & 2.0109 & 1.96 & reject $H_{0}$ & 35 & 87.7673 & 1.96 & reject $H_{0}$ \\
\hline 16 & 2.3067 & 1.96 & reject $H_{0}$ & 36 & 23.3605 & 1.96 & reject $H_{0}$ \\
\hline 17 & 1.8006 & 1.96 & do not reject $H_{0}$ & 37 & 17.4334 & 1.96 & reject $H_{0}$ \\
\hline 18 & 2.1880 & 1.96 & reject $H_{0}$ & 38 & 2.1865 & 1.96 & reject $H_{0}$ \\
\hline 19 & 2.5983 & 1.96 & reject $H_{0}$ & 39 & 1.92016 & 1.96 & do not reject $H_{0}$ \\
\hline 20 & 2.3425 & 1.96 & reject $H_{0}$ & 40 & 1.35962 & 1.96 & do not reject $H_{0}$ \\
\hline
\end{tabular}




\section{Results and discussion}

MST is a subgraph that connects all the variables (nodes) whose total weight, i.e., total distance is minimal. In figure 1 , we present the visualization of corresponding MSTs issued from Pajek. Figure 1(a) is for $\bar{S}$ and figure 1(b) is for $S_{35}$. Those figures show the most important relationship among all currencies in terms of MST. From those figures, it is shown that the MST of $\bar{S}$ differs considerably from $S_{35}$. This justifies our conclusion that the covariance matrix of $S_{35}$ is not stable since its structure differs from the structure of the average of stable covariance matrices, $\bar{S}$. According to those figures, for $\bar{S}$, the most important currencies are $G H S, L K R, T R Y$ and $Z M K$, while for $S_{35}$, the most important currency is $B R L$. Those currencies are important because the number of their connections to others currencies are higher than the others. This means that all the currencies in $\bar{S}$ has been influenced by $G H S, L K R, T R Y$ and $Z M K$ and $B R L$ for $S_{35}$.

To elaborate the above finding more clearly, other information will be presented by using the centrality measures, i.e., degree centrality and betweenness centrality. In figures 2 and 3, we present the network topology where the size and color of the node represent the score of centrality measure and the rank of importance, respectively. The colors used in this analysis, ordered decreasingly in terms of the rank of importance: yellow, red, blue, purple and orange. The higher the score of the centrality measures of a particular node, the more influential that node is. In this study, the most influential node is a node which is responsible for the instability of the covariance matrix.

From figure 2, based on degree centrality, for $\bar{S}, G H S$ has the highest number of connections in the network, i.e., 38. Followed by $T R Y, L K R, Z M K$ and $S A R$ with 10, 9, 7 and 5 connections, respectively. The rests are of 1,2, 3 and 4 connections only. On the other hand, for $S_{35}, B R L$ has the highest number of connections in the network, i.e., 71 . Followed by $A U D$ with 3 connections and $A R S, M X N, P E N$ and XPT have 2 connections for each. The rests are of 1 connection only. The higher the number of connections, the more influential the particular currency is. Manually, the number of connection of a currency is determined by the number of currency that connects to that currency.

In terms of betweenness centrality, see figure 3, for $\bar{S}, G H S$ plays the most important role in the network followed by, in order of importance: TRY, LKR, JMD and ZMK. While, for $S_{35}, B R L$ plays the most important role in the network followed by, in order of importance: $A U D, A R S, M X N, P E N$ and $X P T$. This means that if those currencies are well managed, then the others will be influenced.

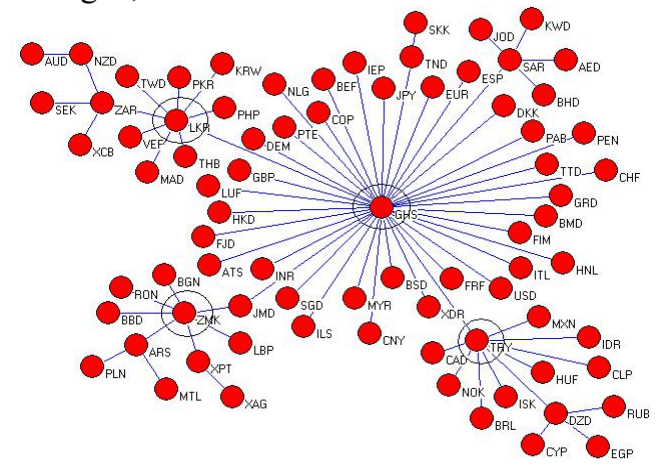

(a) $\bar{S}$

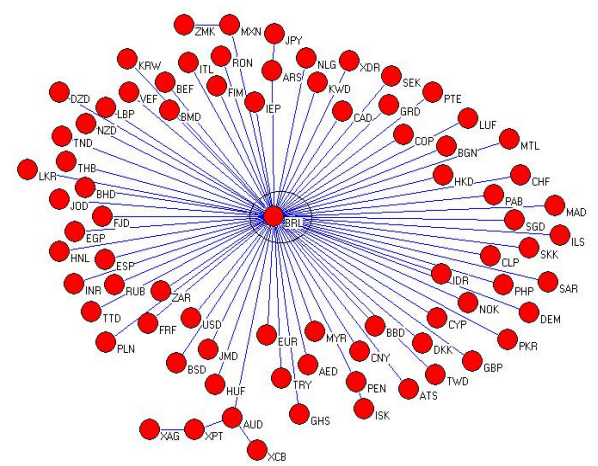

(b) $S_{35}$

Fig. 1. MST of (a) $\bar{S}$ and (b) $S_{35}$. 


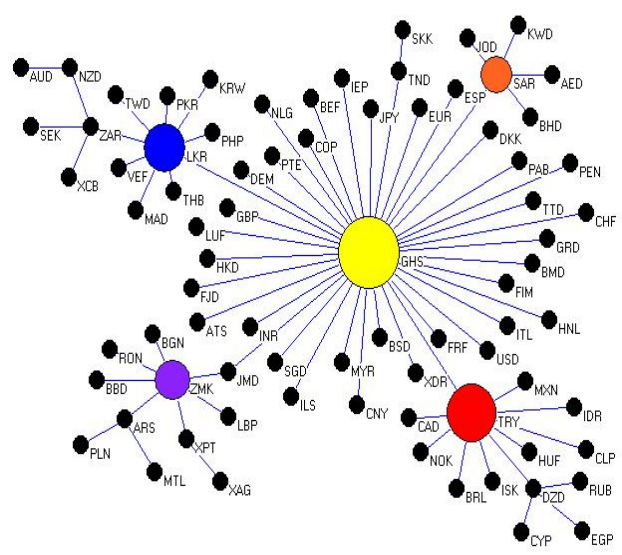

(a) $\bar{S}$

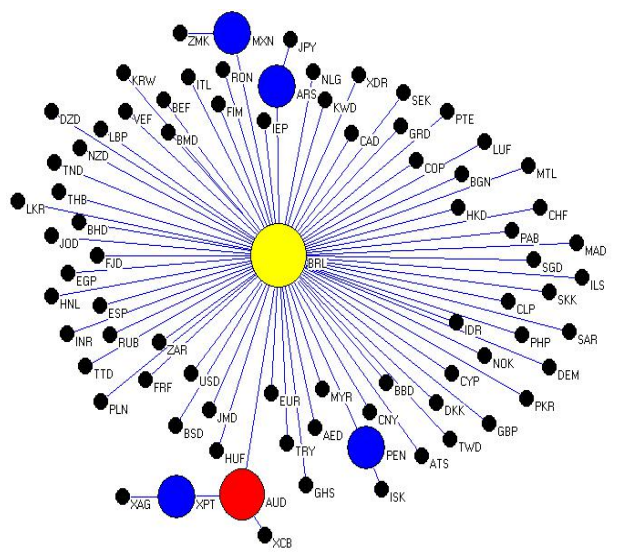

(b) $S_{35}$

Fig. 2. Degree Centrality of (a) $\bar{S}$ and (b) $S_{35}$

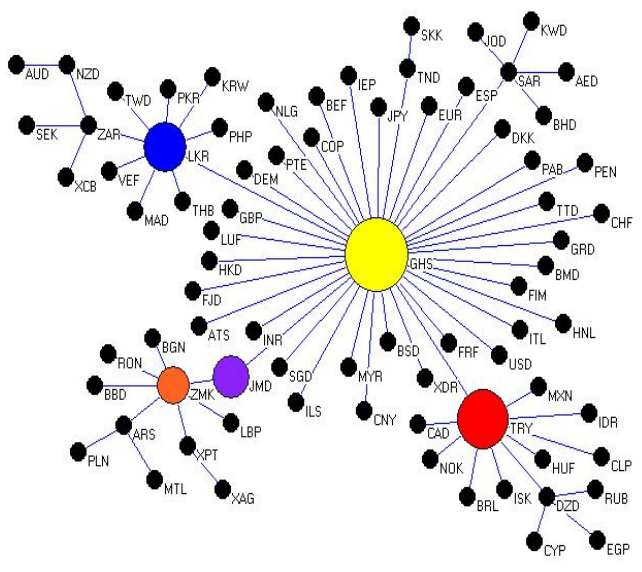

(a) $\bar{S}$

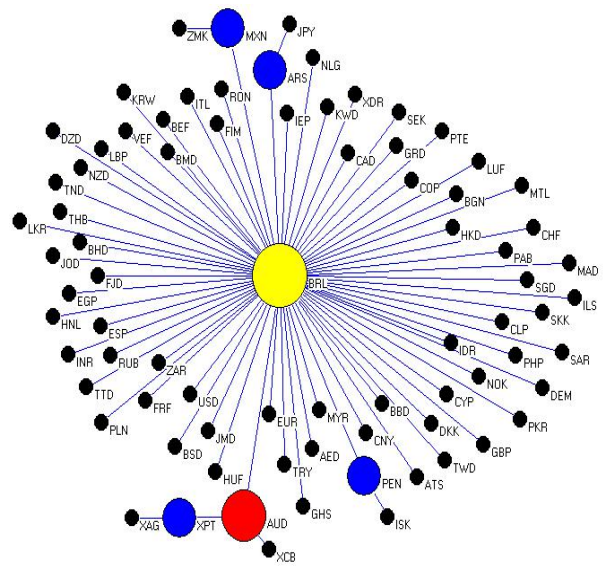

(b) $S_{35}$

Fig. 3. Betweenness centrality of (a) $\bar{S}$ and (b) $S_{35}$.

\section{Conclusion}

Based on the analysis on MST in figure 1, we learn that all the currencies in $\bar{S}$ is influenced by $G H S, L K R, T R Y$ and $Z M K$. On the other hand, for $S_{35}, B R L$ does influence the other currencies. Further analysis based on two centrality measures leads us to the following conclusions.

(i) For $\bar{S}$, the following currencies are among the five highest scores for at least one measure, i.e., the most influential currencies: GHS, JMD, LKR, SAR, TRY and ZMK.

(ii) For $S_{35}$, the following currencies are among the five highest scores for at least one measure, i.e., the most influential currencies : ARS, AUD, BRL, MXN, PEN and XPT.

Therefore, based on these findings, we conclude that the differences between $\bar{S}$ and $S_{35}$ is generally caused by currencies $A R S$ (Argentina Peso), AUD (Australia Dollar), $B R L$ (Brazilian Real), GHS (Ghana Cedi), JMD (Jamaican Dollar), LKR (Sri Lankan Rupee), $M X N$ (Mexican Peso), PEN (Peruvian Sol), SAR (Saudi Riyal) TRY (Turkish Lira), XPT 
(Ounces of Platinum) and ZMK (Zambian Kwacha), i.e., these currencies are responsible for the instability of $S_{35}$. Consequently, these currencies should be given special attention in Foreign Exchange Rate.

\section{Acknowledgement}

We acknowledge financial support from Universiti Malaysia Pahang, via Internal Grant vote number RDU1703268. The authors would like to thank Universiti Malaysia Pahang, Universiti Utara Malaysia and Universiti Teknologi Malaysia Kuala Lumpur for the opportunity to do this research. Special thanks go to the reviewers for the comments and suggestions.

\section{References}

1. Tang G Y N 1998 Applied Financial Economics 8 359-365.

2. Stephen L 2006 Journal of Property Investment \& Finance 24 (5) 434-442.

3. Schindler F 2009 Journal of Property Investment and Finance 27 (6) 579-592.

4. Eichholtz P M A 1996 Journal of Real Estate Research 11 (2) 149-158.

5. Wood M 1994 International Journal of Service Industry Management 5 (4) 53-68.

6. Roes K C B and Dorr D 1997 International Journal of Quality Science 2 (3) 149-166.

7. Djauhari M A 2005 Journal of Quality Technology 37 (1) 32-39.

8. Mason R L, Chou Y M and Young J C 2009 Communications in Statistics-Theory and Methods 38 (6) 939-951.

9. Florac W A, Carleton A D and Barnard J R 2000 IEEE Software 97-106.

10. Jalote P and Saxena A 2002 IEEE Transactions on Software Engineering 28 (12) 1125-1133.

11. Hanslik T, Boelle P Y and Flahault A 2001 Public Health 115 277-281.

12. Beamon B M and Ware T M 1998 International Journal of Physical Distribution and Logistics Management 28 (9/10) 704-715.

13. Yusoff N S and Djauhari M A 2013 Jurnal Teknologi 63 (2) 81-83.

14. Yusoff N S and Djauhari M A 2011 Proceedings of 2011 IEEE International Conference on Quality and Reliability (ICQR) 2011 617-619.

15. Djauhari M A 1994 Proceedings ICCS-VI, Lahore, Pakistan 8 423-428.

16. Gray J B and Ling R F 1984 Technometrics 26 (4) 305-318.

17. Sharif S, Ap N C and Ruslan N 2017 AIP Conf. Proc. 1847020013.

18. Yusoff, N.S. and Sharif S. 2017 Journal of Physics: Conference Series, 890 (1).

19. L. C. Freeman, S. P. Borgatti and D. R. White, Social Networks 13, 141-154 (1991).

20. Yusoff, N.S. \& Shamshuritawati Sharif (2016) Advanced Science Letter, p.p. 1-5. 